Théologiques

Théologiques

\title{
« Dieu » dans la théologie pratique : ressource ou tabou?
}

\section{Jean-Guy Nadeau}

Volume 6, numéro 2, octobre 1998

Dieu interdit

URI : https://id.erudit.org/iderudit/024964ar

DOI : https://doi.org/10.7202/024964ar

Aller au sommaire du numéro

\section{Éditeur(s)}

Faculté de théologie de l'Université de Montréal

\section{ISSN}

1188-7109 (imprimé)

1492-1413 (numérique)

Découvrir la revue

\section{Citer cet article}

Nadeau, J.-G. (1998). « Dieu » dans la théologie pratique : ressource ou tabou? Théologiques, 6(2), 73-98. https://doi.org/10.7202/024964ar

\section{Résumé de l'article}

Le rôle de " Dieu » dans la théologie pratique (TP) varie selon les différentes orientations de la TP. Malgré des recoupements dûs aux chevauchements des types de TP, Dieu apparaît surtout comme objet de croyance et motivation de l'action dans les TP empiriques, alors que les TP socio-critiques l'utilisent comme référence prophétique ou axiologique. Les TP fondamentales le traitent souvent comme symbole discursif, alors qu'il fonctionne comme Sujet convocateur et Parole interpellante dans les TP pastorales. L'article situe ces hypothèses sur le fond de scène des rapports de la TP avec la théologie et les sciences sociales, avant de s'interroger en finale sur le rôle de " Dieu » et de la TP dans un monde " scientifique ". 
Théologiques 6/2 (1998) 73-98.

\title{
" Dieu » dans la théologie pratique : ressource ou tabou?
}

\author{
Jean-Guy NADEAU \\ Faculté de théologie \\ Université de Montréal
}

Si la tradition, les catégories religieuses, et en particulier la justice et la bonté de Dieu, ne sont pas transmises comme des dogmes, comme des vérités absolues, mais comme la nostalgie de ceux qui sont capables d'une vraie tristesse [...] la mentalité théologique, ou tout au moins sa base, pourrait être conservée sous une forme adéquate [...]. Car nous devons tous être liés par la nostalgie que ce qui arrive dans le monde, l'horreur et l'injustice, n'en sont pas le dernier mot, mais qu'il existe un Autre. [...] Nous devons être unis dans la conscience que nous sommes des êtres finis. Nous ne devons pas abandonner le concept d'infini qu'a développé la religion, mais nous ne devons pas en faire un dogme [...]. (HORKHEIMER ${ }^{1}$ )

Ce numéro de Théologiques s'est élaboré à partir de l'impression d'un malaise, voire d'un tabou concernant la nomination de Dieu en sciences humaines et sociales. D'où l'usage des guillemets dans le titre de l'article, guillemets que l'on pourra soupçonner de jouer le rôle d'amulettes face au tabou scientifique entourant ou excluant "Dieu». Or il a semblé à certains que la théologie elle-même n'échappait pas à ce tabou, renouant peut-être, au mieux, avec celui du divin tétragramme dans la tradition juive. D'où cet article sur la place de « Dieu» dans la théologie pratique, place déterminée par le statut "pratique " de la théologie, par sa situation dans le champ des sciences humaines, et par la diversité des théologies pratiques. 


\section{Une théologie «pratique »}

Il y a longtemps que l'on s'interroge sur le statut «pratique " de la théologie. Considérant la question, Thomas d'Aquin remarquait d'abord que la théologie traite de la foi qui implique une activité et donc une science pratique. Toutefois, poursuivait-il, une science pratique traite des actes humains et de ce qui relève de l'homme, alors que la théologie traite principalement de Dieu dont la connaissance est révélée et n'est pas le fruit de l'activité humaine. L'Aquinate en concluait que la théologie, la doctrina sacra, était « plus spéculative que pratique " (Somme théologique I, Q.1, art. IV). On dira donc que la dimension spéculative de la théologie accorde à Dieu une place centrale soit comme objet de quête, de connaissance et de discours, soit comme sujet même d'une théologie qui en déploie l'action révélante. Par contre, l'humain serait l'objet de la dimension pratique de la théologie et Dieu n'en serait qu'un objet indirect, connaissable tout au plus par la relation que les humains entretiennent avec lui et qu'ils mettent en pratiques et en discours.

L'objet direct d'une théologie qui se veut pratique ne sera donc pas Dieu, «but rather human experience of God, our communication with Him and about Him. God can be only an indirect object and ultimate goal." (VAN DER VEN 1993 : 29; et 1988). Si cette affirmation de Johannes van der Ven, vise d'abord la "théologie empirique ${ }^{2}$, comme il désigne la théologie pratique, ses références à Schleiermacher, R. R. Niehbur et Schillebeeckx, de même - mais avec le signe contraire - qu'à Hegel, Barth et H. R. Niehbur, indiquent qu'elle concerne l'ensemble de la théologie ${ }^{3}$.

Une large part de la théologie contemporaine, particulièrement en contexte universitaire, $s$ 'inscrit dans cette perspective qui va à l'encontre de l'image de la théologie véhiculée par les sciences humaines qui, confondant théologie et dogmatique, se demandent si Dieu (et sans doute Dieue) peut être objet de savoir et de recherche scientifiques ou universitaires. C'est ainsi que des théologiens en

2 Étrange combinaison que cette "théologie empirique ", disent certains. Voir TILLICH (1967: 40-46) et AUDINET (1995: 249).

3 Déjà ZAHRNT (1971:129ss) utilisait le terme pour désigner une théologie de la pratique et de l'expérience chrétienne à la lumière de la Révélation. 
sont venus à opter pour une théologie comme grammaire ${ }^{4}$, une théologie qui soit "la description de la façon dont nous parlons de Dieu, et non plus l'étude des mots qui renvoient à un objet mystérieux " (VIAU 1993 : 143), encore moins l'étude de cet « objet » lui-même.

Enfin, plusieurs théologiens et théologiennes soutiennent, du côté protestant depuis Luther et aujourd'hui du côté catholique, que toute théologie est pratique 5 . Soit, pour certains, parce qu'elle porte sur l'agir de Dieu dans l'histoire, particulièrement en Jésus Christ. Soit, pour la plupart, parce qu'elle porte sur la foi qui est expérience humaine, acte d'existence. Heidegger (1969: 379) avait bien saisi cette dynamique qu'il exprimait ainsi à des théologiens de Tübingen :

Puisque la Théologie comme discipline tant systématique qu'historique a pour objet premier l'événement chrétien dans sa christianité et dans son historicité, puisque cet événement se définit comme mode d'existence du croyant, et que d'autre part exister est agir, est praxis, la Théologie a donc de par son essence le caractère d'une science pratique. Comme science de l'action de Dieu en l'homme agissant dans la foi, elle est de soi déjà homilétique. C'est uniquement pour cela qu'il est possible que la théologie dans son organisation de fait se constitue elle-même comme Théologie pratique, comme Homilétique et Catéchétique, et non pas parce que des besoins fortuits exigent que des propositions en soi théoriques aient aussi une application pratique. $\mathrm{La}$ Théologie n'est systématique que lorsqu'elle est historico-pratique. Elle n'est historique que lorsqu'elle est systématico-pratique. Elle n'est pratique que lorsqu'elle est systématico-historique 6 .

Si toute théologie est pratique, saisir Dieu dans le champ de la théologie pratique équivaudrait à le saisir dans le champ de la théo-

4 Après Luther et Wittgenstein, voir KERR (1991: 199ss).

5 AUDINET (1977), EICHER (1982), GISEL (1975: 603), GREINACHER (1990), KINAST (1983), LUCIER (1987), METTE (1978 et 1979), OGLETREe (1983), PEUKERT (1976), VIAU (1993), etc. Constatant le fait, DUQUOC en parle comme d'une prise de pouvoir et d'une dérive (1996: 1071). Signalons que Duquoc utilise, lui aussi, la typologie d'Audinet dans sa présentation de la TP.

6 Les italiques sont de Heidegger. 
logie. Ce n'est pourtant pas en ce sens que nous parlerons ici de la théologie pratique, mais plutôt, dans son acception technique, comme d'une discipline théologique déterminée par son rapport critique à la pratique (aussi discursive) des croyants. Mais, avant d'en venir à cette théologie pratique, quelques remarques sur la solitude du discours théologique dans le champ du savoir dresseront la toile de fond de notre réflexion.

\section{La solitude du discours théologique dans la rationalité contemporaine}

Dans son ouvrage sur La nouvelle théologie pratique, Marcel Viau (1993 : 276) a cette belle formule selon laquelle "les mots utilisés par le théologien se présentent au front de l'objet comme un soldat solitaire " 7 . Parmi ces mots, Dieu est sans doute le plus délicat. Or, ce n'est peut-être pas tant le mot "Dieu " lui-même qui fait problème, mais la portée auto-implicative du discours théologique et surtout la prétention traditionnelle (et parfois contemporaine) de la théologie à " parler de Dieu ».

Le tabou de Dieu s'inscrit sur le fond de l'épistémè empirique des sciences sociales. Réfléchissant à leur rapport à la théologie, Benoît Garceau (1989) rappelle qu'elles se sont développées dans une visée anti-théologique (particulièrement, préciserons-nous, quant à l'autorité, au dogme et à la métaphysique). Deux postulats fondamentaux déterminent l'opposition épistémologique des sciences sociales vis-àvis de la théologie : l'idée que la connaissance doit être basée sur une observation empirique déterminante pour la vérité des énoncés; la conscience de l'historicité de la connaissance et de notre rapport à l'univers. Signalons par ailleurs que ces postulats marquent aussi la théologie contemporaine, quoique de façon variable, et particulièrement la théologie pratique.

Malgré les nouvelles épistémologies herméneutiques, néo-prag matistes et constructivistes qui interrogent les prétentions "réalistes " des sciences humaines, le domaine du savoir (pratiquement réduit à ce qui est publiquement observable) et celui du croire s'en trouvent radicalement dissociés. On peut croire en Dieu, et les

7 Pour un débat autour de cette Nouvelle théologie pratique, voir NADEAU et VIAU (1996). 
sciences sociales sont prêtes à étudier les phénomènes de la foi et de la croyance, mais peut-on en savoir quelque chose? La logique des sciences sociales s'avère sensible aux mécanismes de production des pratiques et du discours religieux, parfois à leur sens pour l'humain, mais non à la vérité des énoncés sur Dieu à laquelle prétend la théologie. Ainsi considèrent-elles que la religion, et par conséquent la théologie, ne peut parler que de l'homme et non de ce qui est (ou serait) extérieur à l'humain.

La ratio théologique classique apparaît alors comme un vestige du passé, sans pertinence face aux rationalités et aux discours contemporains. Le discours théologique, qui présuppose encore largement un sens révélé, s'en trouve perçu comme inadéquat à décrire la réalité, disqualifié et même poussé vers l'insignifiance (VIAU 1993 : 13), alors que son concept clé est devenu tabou, échappant à la sécurité du champ scientifique. Dieu n'est pas une hypothèse de travail pour les sciences sociales: il ne relève ni de leur champ d'observation ni de leurs méthodes d'appréhension, et ne trouve donc pas place dans leurs théories, si ce n'est le plus souvent comme construit aliénant. Par contre, le sujet humain de la théologie et le projet théologique de connaissance peuvent trouver pertinence aussi bien en sciences humaines qu'en théologie.

L'objet de la théologie aurait donc changé, ce dont la théologie pratique a pris acte d'une façon particulière. Si plusieurs justifient cette évolution de l'intérieur même de la théologie, d'autres la considèrent comme le fruit de l'interdisciplinarité en théologie, le dialogue avec les sciences humaines faisant éclater la définition même de la théologie. Si d'aucuns s'en désolent, y voyant une perte d'identité de la théologie, d'autres y voient un progrès pour la théologie que les nouvelles rationalités des sciences humaines auraient amenée non seulement à transformer mais à développer et à renforcer son identité disciplinaire (FORTIN-MELKEVIK 1994: 132). D'une part, en lui fournissant des instruments adéquats pour appréhender l'expérience, le langage et les pratiques qui constituent non seulement des objets de la théologie (pratique) mais définissent des conditions de possibilité même du discours théologique. D'autre part, en ce que la théologie ne se comprend plus comme une discipline à part, mais au milieu d'autres disciplines et soumise aux mêmes chocs épistémologiques dont elle ne peut faire abstraction pour se constituer en rationalité contemporaine. 
La légitimité de la théologie, poursuit Anne Fortin (pp. 135. 136), ne peut " plus se déduire a priori en fonction d'un sens et d'une vérité possédés de toute éternité [mais elle est] à construire dans un dialogue interdisciplinaire devant rendre compte de ses transformations épistémologiques devant des sujets croyants, devant la communauté. »Par exemple, l'herméneutique, l'analyse structurale, la pragmatique, en faisant éclater la prétention d'un accès privilégié à un sens pré-donné et à la vérité, en saisissant le sujet comme «sujet interprétant dont la question joue un rôle déterminant dans le dévoilement du sens ", ont permis à la théologie de redéfinir sa ratio en termes interprétatifs (p. 136). La théologie est alors en mesure de se distinguer aussi bien du paradigme métaphysique et déductif de la théologie classique que du paradigme empirique des sciences sociales.

Mais l'évolution de la place de Dieu en théologie n'est pas liée seulement à ses rapports avec les sciences humaines et sociales. Elle l'est tout autant, me semble-t-il, à la perte de contrôle, voire d'influence, du magistère ecclésiastique sur la théologie, particulièrement sur la théologie universitaire. Ce nouveau statut institutionnel de la théologie, qui a facilité le passage d'une théologie pratique d'application pastorale à une théologie pratique de recherche et d'élaboration critique, questionne vivement une idéologie commode et encore trop répandue qui fait de la théologie la courroie de transmission du magistère romain. On peut cependant se demander si la théologie, du moins en contexte universitaire, n'a pas remplacé son inféodation au magistère et aux projets ecclésiastiques, par une dépendance aux organismes subventionnaires tels, au Québec, le $\mathrm{CRSH}$ et le FCAR où siègent justement des praticiens d'autres disci plines des sciences humaines et sociales qui font aujourd'hui autorité et poursuivent leurs propres projets.

Voyons les conséquences de cette évolution sur la place de Dieu dans la théologie pratique.

\section{La place de Dieu dans la théologie pratique}

\subsection{La théologie pratique}

Les définitions de la théologie pratique sont multiples et recouvrent souvent celles d'autres disciplines théologiques. Presque toutes 
en font une théorie critique de la pratique ecclésiale et souvent des autres disciplines théologiques 8 dont elle constitue la "fine pointe " (JÜNGEL 1968: 40) ou la " couronne " (SCHLEIERMACHER, 1988 : 99; et 1994). D'autres la considèrent comme une discipline " concernée par l'étude de structures sociales spécifiques et d'initiatives individuelles dans lesquelles devient manifeste l'œuvre divine continue de renouveau et de réparation, que l'on peut trouver à l'intérieur ou à l'extérieur de la vie ecclésiale. " (CAMPBELL 1990: 18) Schillebeeckx considère que son objet formel " est de rapprocher [de façon scientifique et critique] les intuitions théologiques et les faits empiriques d'une manière qui soit méthodologiquement fondée et systématiquement organisée "(VAN DER VEN 1994 : 112-113). Pour la majorité, dont un théologien systématique se fait l'écho, elle est " une théologie qui non seulement part de la pratique comme lieu théologique, mais est prête à se laisser mettre en question par la pratique " (GEFFRÉ 1983: 343), une théologie pratique réflexive saisie comme théorie scientifique au cœur de l'action (RAHNER 1970: 113, NIPKOW 1993 : 51). C'est ainsi que la TP « accorde à la praxis [...] une primauté sur la théorie aussi bien au plan épistémologique (comme source de connaissance) qu'au plan téléologique (la transformation de la pratique elle-même). Son parcours méthodologique [va] de la praxis initiale à la praxis transformée par la médiation indispensable d'une ou de plusieurs théories. " (MÉNARD 1992 : 300; voir BOFF, 1990) On le voit, les rapports de la TP à la pratique, dont le concept reste souvent mal défini, sont multiples et variés.

Ces théologies présentent néanmoins certains dénominateurs communs. Dans une réflexion sur la TP américaine, Mary-Elisabeth Moore (1995:2) constate par exemple que celle-ci, malgré sa diversité, est marquée par trois préoccupations communes: " que la réflexion théologique soit centrée sur les données de l'expérience concrète et de l'action; que les construits théologiques soient, au moins en partie, façonnés par ces expériences et actions; et que la réflexion théologique contribue à la compréhension de l'expérience et à la direction de l'action. » 794), GREINACHER $(1990: 281)$. 
Quant à nous, nous considérons les éléments suivants, dont nous avons déjà présenté certains dans un article antérieur (NADEAU 1990), comme constitutifs de la TP :

- la saisie de l'expérience et de la pratique comme point de départ et point d'arrivée de la réflexion;

- une approche critique de cette expérience ou pratique;

- un rapport étroit avec les sciences humaines et sociales nécessaires à la saisie tant de l'expérience que des pratiques concrètes;

- une démarche interprétative marquée par la mémoire chrétienne, particulièrement par l'Évangile.

Ces éléments nous conduisent aux perspectives de théologie pratique - empirique, critique et fondamentale - identifiées par Jacques Audinet (1995), un pionnier de la TP contemporaine. Dans la mesure où ils marquent presque toute $T P$, ils nous avertissent aussi de la difficulté d'en dresser une typologie; par exemple, approches empiriques et approches critiques se rejoignent souvent bien que leurs objectifs puissent diverger. La typologie d'Audinet en effet ne va pas sans chevauchements ou combinatoires, comme il le signale lui-même (p. 247). Van der Ven, par exemple, désigne comme «empirico-critique » ou "herméneutico-empirique » et normative $(1990 ; 1994 ; 1993: 64-65)$ son approche qu'Audinet qualifie simplement d'empirique, à partir cependant de l'appellation que van der Ven donne à son projet. De même, Audinet classe la praxéologie pastorale parmi les TP fondamentales. Or, cette "science de l'action pastorale » (NADEAU 1990 et 1993) relève aussi des autres types de TP. Elle est empirique dans son analyse des pratiques concrètes; critique dans son rapport à ces pratiques, à l'Église et à la société; fondamentale dans son souci de définir la pastorale ou les conditions d'efficacité des pratiques pastorales; programmatique dans son moment d'opérationnalisation d'une intervention. Quoi qu'il en soit, la typologie d'Audinet met bien en lumière des orientations majeures ainsi que des enjeux et problèmes des diverses TP. Nous l'utiliserons donc pour encadrer notre réflexion sur la place de Dieu en théologie pratique ${ }^{9}$.

9 Audinet s'est intéressé à la référence théologique de ces types de TP, mais dans une orientation différente que celle que nous poursuivons ici. 


\subsection{Quatre hypothèses}

Il eut été intéressant de relire quelques ouvrages récents de théologie pratique et d'y identifier, de façon empirique justement (voire quantitative aussi bien que qualitative), la place qu'ils accordent à Dieu. Mais cela ne nous a pas été possible bien que nous ayons regroupé à cette intention quelques ouvrages importants. On considérera donc ces pages comme une première approche de la question qu'il faudra documenter davantage ${ }^{10}$. Nos hypothèses sont fondées sur une fréquentation non seulement de la littérature de théologie pratique, mais aussi de congrès de sociétés nationales et internationales de théologie pratique (où on ne parle pratiquement pas de Dieu), de même que sur plusieurs années de recherche et d'enseignement en la matière, souvent en team teaching.

En bref, et il faut considérer ces énoncés comme des hypothèses et tenir compte des chevauchements entre les types de TP, Dieu est surtout présent dans les TP empiriques comme objet de croyance et motivation de l'action. Il apparaît comme la référence prophétique ou axiologique ultime des TP critiques, alors que les TP fondamentales le traitent comme occurence langagière ou symbole discursif. Une quatrième hypothèse concerne les TP pastorales qui visent l'élaboration de programmes, auxquelles Audinet ne fait pas allusion, et où Dieu fonctionne comme Sujet convocateur et Parole interpellante. Voyons cela de plus près.

\subsection{Les théologies pratiques empiriques}

La recherche empirique ou, à tout le moins, le rapport à l'empirie (le plus souvent prise pour acquise et non définie) caractérise la TP. "Point de théologie pratique qui ne soit empirique par un pôle. Point de théologie pratique qui soit seulement empirique. "(AUDINET 1995 : 250) Par ailleurs, certaines approches de TP auscultent la réalité avec plus de rigueur méthodologique que d'autres et ne portent pas de programme politique ou théologique particulier. Ce sont ces TP qu'Audinet qualifie d'empiriques. Elles s'intéressent à l'analyse et à l'évaluation des pratiques religieuses ou, plus largement, à l'ana-

C'est ainsi que nos références ne prétendent à aucune exhaustivité mais simplement à illustrer nos propos. $\grave{A}$ ce titre, elles ne pourront malheureusement faire justice à ceux et celles qui œuvrent en TP. 
lyse du fait religieux dans la société. Elles recourent aux outils habituels d'enquête quantitative ou qualitative: étude de mentalités, sondage d'opinion, évaluation de projet (parfois dans une approche quasi expérimentale), interview, analyse de contenu, recherche-terrain, analyse de politiques, etc. Ces recherches comportent souvent un souci de pertinence de l'agir ecclésial ou pastoral convoqué à en utiliser les résultats. Elles portent aussi un enjeu politique général dans la mesure où la connaissance de leurs résultats redonne aux acteurs du pouvoir sur leur situation ou leur pratique.

Actuelle figure de proue de la TP empirique et directeur du Journal of Empirical Theology, Johannes van der Ven signale deux modalités principales de ces recherches quant au rapport aux sciences sociales. Soit que le chercheur en TP utilise les résultats d'enquêtes menées en sciences sociales, mais cela n'est pas l'idéal dans la mesure où il se trouve alors à la remorque de leurs préoccupations et de leurs grilles d'analyse. Soit, et c'est la position privilégiée par van der Ven, qu'il mène lui-même l'enquête (si possible avec d'autres), ce qui lui permet d'y inscrire dès le départ une dimension ou des préoccupations théologiques.

\subsection{Dieu, objet de croyances et item d'enquêtes}

Les analyses de pratiques, on s'en doute, font peu de place à Dieu. Je me rappelle ces questions d'étudiants en praxéologie pastorale qui demandaient s'ils pouvaient inclure Dieu (ou l'Esprit Saint) parmi les acteurs des pratiques qu'ils étudiaient. L'étude se voulant empi rique, la réponse était évidemment négative. Certes, on peut identifier la foi en Dieu comme moteur ou justification idéologique de l'action de certains sujets, et en tenir compte à ce titre. Mais comment considérer au plan empirique un acteur transcendant dont on ne saurait identifier l'action propre, un acteur dont la théologie affirme massivement qu'il n'agit qu'à travers les humains? D'où la mise entre parenthèses, l'épochè que les TP empiriques font, parfois à regret, de Dieu comme sujet ou acteur de l'histoire, qualité divine pourtant fondamentale pour la foi (et la théologie).

Dieu est par contre très présent dans les enquêtes sur les croyances, et dans certaines recherches évaluatives, par exemple sur l'éducation religieuse. Il y apparaît comme objet de croyance, un item parmi d'autres, bien que fondamental dans une recherche théo- 
logique, dont il s'agit de repérer et de qualifier la place dans les croyances des sujets de l'enquête et, éventuellement, l'influence sur leurs pratiques. De là, on cherchera par exemple à comparer entre eux différents concepts de Dieu portés par les croyants ou la population. Ces recherches sont donc cohérentes avec la notion que Dieu n'est pas objet de la théologie, mais plutôt la foi.

À l'évidence, Dieu ne saurait être objet d'analyses empiriques. Ce qui l'est, ce sont les pratiques et les discours religieux, ou encore le rapport des pratiques et discours séculiers avec diverses croyances religieuses plus ou moins explicites. La TP empirique ne prétend pas parler de Dieu - et en cela elle échappe au défi du discours sur Dieu -, mais des différentes perceptions qu'en ont les croyants et des effets de ces perceptions. Plusieurs signalent cependant le risque que la théologie se transforme en sociologie ou en psychologie de la religion, en science de l'action religieuse, etc. D'où la nécessité de la phase interprétative des TP empiriques, phase qui permet de les qualifier de théologiques aux yeux de plusieurs.

Dieu est autrement présent dans cette phase interprétative qu'il ne l'était dans les phases précédentes. On se trouve alors devant deux possibilités majoritaires. Dans la première, le concept de Dieu paraît déterminant à cause des sources bibliques ou théologiques utilisées comme références et matrices de ces interprétations. Apparaît alors le problème de l'articulation, voire de "l'impertinence réciproque " (Lalive D'EPINAY 1978) ${ }^{11}$ de données empiriques et de données révélées, de même que celui, non plus résolu, de l'autorité respective de ces données. ${ }^{12}$ Dans la seconde possibilité, la phase interprétative des recherches de TP empirique repose sur une perspective normative et critique. Il y a cependant problème lorsque l'interprétation est fondée sur des axiologies révélées dont Dieu est tenu pour garant, la recherche échappant alors à la perspective herméneutico-empirique prônée par van der Ven (1993: 65). Le problème de la place de Dieu dans la phase interprétative des TP empiriques repose alors sur l'idée (plus ou moins critique, herméneutique ou fondamentaliste) de révé-

11 La pertinence réfère ici à l'adéquation du système composé par l'observateur, sa théorie, son appareillage et son champ d'observation.

12 Sur cette question, voir VAN DER VEN (1994); NADEAU et FOUCAULT (1992). 
lation et sur l'usage, en principe normatif, de cette idée et de son contenu. Nous y reviendrons.

\subsection{Les théologies pratiques critiques}

Les TP critiques, en fait socio et ecclésio-critiques ${ }^{13}$, relèvent d'une théologie engagée dans la transformation de l'Église et du monde. Audinet signale que leurs bases sont multiples et en interaction constante : base théologique issue de la tradition prophétique, base philosophique issue de la théorie critique (que l'on retrouve aussi dans la TP empirique de van der Ven), bases méthodologiques empruntées à la sociologie critique et à l'analyse institutionnelle. Si ces TP comportent une dimension empirique, celle-ci est à l'évidence subordonnée à leur dimension critique, à une axiologie et à un programme qui affirment nettement leurs couleurs. L'éthique et, dans certains cas, l'ecclésiologie constituent une dimension majeure de ces théologies dont les analyses et les évaluations sont subordonnées à des conceptions du vivre en société et de la mission de l'Église dans cette société. Ces TP ont donc une très forte portée évaluative des pratiques et institutions sociales aussi bien qu'ecclésiales ${ }^{14}$.

Cette perspective critique est si présente en TP que Poling et Miller (1985: 29-61) l'inscrivent sur les deux axes de leur typologie des TP américaines ${ }^{15}$. Le premier axe, celui de la méthode, réfère à l'objectivation de la tradition chrétienne par une méthode critique ou une discipline séculière. Le second axe, celui de l'objectif, réfere quant à lui à la mise en relation plus ou moins critique de l'Église et de la société.

Longtemps directeur de la section "Théologie pratique » de la Revue internationale de théologie Concilium, Norbert Greinacher qualifie, comme van der Ven, la TP de méthodique, empirique, her-

13 J'ajoute cette précision étant donné le caractère critique de la TP elle-même, comme le démontre bien la typologie de Poling et Miller (voir plus bas).

14 SCHLEIERMACHER (1988: 108) considérait par exemple que la théologie pratique doit pouvoir servir à évaluer les méthodes que nous utilisons dans l'Église.

15 On trouvera dans VIAU (1987: 72-75), un bon résumé de la typologie de Poling et Miller. 
méneutique et normative. Mais il l'identifie comme théologie prophétique et théorie critique. Elle comporte pour lui trois éléments constitutifs : l'analyse de la situation contemporaine, la cause de Jésus, la pragmatique comme science et orientation éthique de l'action.

Comme théologie prophétique, la TP implique le passage d'une théologie politique classique légitimant l'organisation et les structures politiques actuelles, à une théologie prophétique qui met en cause la situation présente. Si la théologie tente, à partir du fondement biblique, de réfléchir de nos jours à la réalité de Dieu, au monde et aux humains, elle implique nécessairement pour Greinacher une critique impitoyable de la situation politique et économique actuelle. Il s'agit de transformer la situation sociale et ecclésiale pour frayer un passage à l'avènement du Royaume : "Nous ne prenons Dieu et son royaume au sérieux que si nous acceptons de transformer la situation présente. " (GREINACHER $1990: 285$ ) C'est le même souci que l'on retrouve chez les prophètes de l'Ancien Testament, Jésus, les théologies contextuelles, les théologies de la libération et les théologies féministes.

Comme théorie critique, la TP se situe dans la foulée de l'école de Francfort, qui considère la philosophie comme une activité pratique, politique et émancipatrice ${ }^{16}$. Adoptant ce programme, la TP se définira en termes de critique et de transformation sociales, mais avec une visée particulière envers la praxis de l'Église dans le monde. Inscrite dans les paramètres de la théorie critique, la TP s'interrogera non seulement sur la fidélité de la praxis ecclésiale avec la cause de Jésus portée par l'Évangile, mais aussi sur ses rapports avec les prises de conscience de l'histoire récente des libertés. Ainsi Greinacher $(1990: 286)$ précise-t-il que la TP ne s'occupe pas seulement de l'Église et de sa pratique, mais du salut, du bonheur et de la liberté de l'ensemble des humains. La théologie de la libération et la théologie pratique féministe, dont la base empirique est souvent importante (BARONI et all. 1995) relèvent à n'en pas douter de cette mouvance. Particulièrement soucieuse du sort des minorités et

16 Sur la pertinence de la théorie critique pour la théologie pratique, bien que l'auteure n'aborde pas comme telle, on lira avec profit HEWITT (1995). 
des opprimés, la TP s'avère à son mieux agent de changement religieux et social.

\subsection{Dieu, fondement de la théorie critique}

De par leur fondement prophétique, la majorité des TP critiques semblent attribuer à Dieu un rôle de référence ultime, voire de fondement de leur théorie critique, leurs concepts analytiques fussent-ils empruntés à l'École de Francfort. Même si ces théologies recourent à la recherche et à l'analyse empiriques, même si elles se réfèrent massivement aux concepts opératoires et aux méthodes des sciences humaines, elles sont portées par des interrogations qui touchent aux «desseins de Dieu " aussi bien qu'aux projets humains et dépassent les critères empiriques ou même éthiques. Le parcours, précise un Audinet qui a lui-même mené ce type de travail, va alors de l'analyse de l'institué des formes sociales et ecclésiales à l'instituant du salut de Dieu parmi les hommes (1995: 250).

On peut cependant se demander si la référence à Dieu joue bien comme fondement réel de ces théologies ou si elle n'opère pas aussi comme caution divine ou justification théologique, voire idéologique, d'un fondement critique séculier. En poussant encore plus loin le soupçon, et sans doute de façon injuste, on peut aussi se demander si cette référence ne joue pas comme sacrifice du discours critique à une transcendance qui paraît plus que jamais échapper à nos pratiques. Y trouverait-on à la limite un rite discursif remplaçant chez certains théologiens des rites liturgiques tombés en désuétude?

Ainsi en est-il, par exemple, lorsqu'on réfère à la pratique de Jésus ou au dessein, voire au jugement de Dieu pour fonder le droit de tous à la justice et les pratiques de solidarité afférentes à ce droit. Autre exemple: l'abus sexuel des enfants est devenu dans certaines pratiques et discours sociaux, voire scientifiques, l'équivalent du péché mortel dans le christianisme. Des réflexions de TP sur la question la référent à la Bible ou au dessein de Dieu sur l'humanité. Mais avons-nous besoin de la Bible ou d'une caution divine pour nous scandaliser de ces abus et y réagir?

Par ailleurs, si la référence divine n'est pas toujours nécessaire au discours critique, il y a des cas où elle s'avère plus qu'un rite destiné à dédouaner le théologien quant à sa foi, sa communauté ou son Dieu. Les théologies de la libération et les théologies féministes illustrent 
bien ces cas où une référence théologique s'avére nécessaire lorsque des concepts théologiques servent à justifier auprès des croyants des pratiques ou des institutions injustes et oppressives. C'est alors de l'intérieur du système de foi ou du système de croyances que doit se faire la critique et non seulement de son extérieur. En ce cas, Dieu est pertinent dans le discours critique parce qu'il l'était déjà dans la "réalité ", le discours institué ou le système de croyances. Respectant face aux croyants le principe de charité, c'est-à-dire l'attitude consistant à tenir le plus longtemps possible pour vrai dans les circonstances, que Davidson considère, après Wilson et Quine, comme "la base de l'interprétation " (1993 : 246; voir 14 et 224), la TP peut faire preuve d'une fécondité particulière par rapport aux sciences sociales qui ignorent ce principe quant aux croyants religieux qu'elles soumettent d'emblée à leurs modèles excluant Dieu ou la transcendance.

\subsection{Les théologies pratiques fondamentales}

Les TP fondamentales s'inscrivent sur trois volets majeurs. Certaines s'intéressent à la nature ou aux méthodes de la TP (par exemple, BROWNING 1983; VISSCHER 1990; REYMOND et SORDET 1993). D'autres s'interrogent sur les raisons d'être et les modalités d'une pratique chrétienne ou ecclésiale et s'efforcent d'en élaborer une théorie critique (par exemple, GROOME 1991). D'autres enfin, souvent près de la théologie fondamentale, réfléchissent à la situation de la TP dans le champ du savoir, théologique ou autre. Ces dernières interrogent tant la pratique que le discours chrétiens en fonction de réflexions sur la praxis et la pragmatique, sur les rapports entre théorie et pratique et entre science et théologie, sur l'utilisation théologique des instruments scientifiques d'analyse et les effets de leurs présupposés épistémiques, sur les rapports entre les discours scientifiques et la visée théologique qui commande la motivation et la finalité de la TP, etc.

Cette TP fondamentale est particulièrement sensible au manque d'unité discursive fondamentale en TP. Si l'ecclésiologie jouait auparavant ce rôle en TP, comme la métaphysique en théologie, il en va autrement depuis l'éclatement du champ théologique sous l'impact des sciences humaines La TP recherche alors cette unité, parfois saisie comme fondamentale, du côté de l'anthropologie religieuse (AUDINET), de la connaissance et du langage (VIAU), de la 
communication (VAN DER VEN) de l'esthétique (GRÖZINGER) ou d'une praxis dont relèvent langage et communication (vg. PEUKERT, GROOME, NADEAU).

\subsection{Dieu, référence ultime ou occurence langagière}

Dieu apparaît le plus souvent comme fondement, plus ou moins critiqué, dans les TP qui s'interrogent sur les raisons d'être des pratiques chrétiennes ou de la pratique ecclésiale. Il est moins présent, et c'est alors souvent dans des mises au point qui l'excluent comme objet, dans celles qui s'intéressent à la nature ou aux méthodes de la TP.

Son statut est plus discuté dans les TP fondamentales qui lient les questions de Dieu et de la praxis chrétienne à celles de l'expérience, de la connaissance, du langage, de la communication, de la référence, etc. Dieu y apparaît souvent comme un concept, un objet de croyance ou un terme langagier parmi d'autres, soumis aux mêmes fonctions, aux mêmes aléas, aux mêmes déconstructions. Chez Viau, par exemple, il apparaît comme "l'énoncé placé à la droite de la fonction épistémique " je crois... " (VIAU 1993 : 281), un mot dont la signification n'est pas extra-discursive, mais « surgit des interactions langagières d'un locuteur qui adopte certains comportements. La seule façon pour un organisme de croire en l'existence de Dieu, poursuit Viau, c'est d'envisager le mot Dieu à l'intérieur d'une interaction langagière dans un environnement donné » (VIAU 1993: 276). Mais s'agit-il toujours de théologie pratique ou sommes-nous alors en théologie fondamentale?

Ainsi, dans une recherche sur la fonction révélante des pratiques pastorales (NADEAU 1993b, 1994a, 1994b), je me suis intéressé au rôle de la communication et particulièrement de l'imagination dans la compréhension des pratiques pastorales et dans la révélation (saisie comme adresse à une imagination qui s'ouvre plutôt qu'à une volonté qui se soumet (RICCEUR 1977 : 54). Bien que son souci des pratiques religieuses permette d'identifier cette réflexion comme recherche de TP fondamentale, elle relève peut-être davantage, comme celle de Marcel Viau, de la théologie fondamentale ellemême. 


\subsection{Théologies pratiques pastorales ou programmatiques}

À ces approches de TP empiriques, critiques et fondamentales, j'ajouterais les TP pastorales qui visent le développement de programmes ecclésiaux ou d'interventions pastorales. Si la théologie pratique s'est largement distanciée d'une TP d'application, dont Schleiermacher (1988: 84) rejetait déjà le concept au début du 19e siècle 17 , ou d'une théologie pastorale encore récemment identifiée à la formation pratique des ministres, elle garde le souci d'opérationnaliser les résultats de ses recherches et discours. Ainsi, van der Ven reconnaît à la TP empirique une préoccupation envers le travail des agents de pastorale auxquels elle peut fournir des données aussi justes que possible sur les situations auxquelles ils font face. De son côté, la TP critique interpelle aussi bien l'intelligence, le cœur et l'agir des agents ecclésiaux, comme le fait la TP fondamentale, que celui des agents sociaux.

Par ailleurs, il faut se garder de considérer la TP pastorale comme le moment d'application des types de TP précédents. D'une part, parce que ceux et celles qui y travaillent, surtout en milieu universitaire, s'emploient aussi aux autres types de TP, dans un va-etvient fécond entre l'un et l'autre. D'autre part, parce qu'elles ont souvent leur point de départ propre, enraciné dans la politique ou les priorités d'une communauté particulière. Don Browning illustre bien ce fait dans sa Fundamental Practical Theology qu'il clôt par une «strategic practical theology ». Quoiqu'il en soit, certains verront dans la TP pastorale le test de vérité de la TP elle-même : de quelle utilité, de quelle pratique serait la TP si elle ne peut convaincre et orienter l'agir des agents ecclésiaux qui partagent a priori ses fonde. ments religieux?

On trouve de beaux exemples de TP pastorale dans des ouvrages, par exemple en catéchétique, en liturgie, en animation des communautés, qui présentent leurs propositions de programme ou d'intervention à partir de recherches historiques, théologiques et anthropologiques. On en trouve aussi dans des documents ecclésiaux qui accompagnent leurs programmes de réflexions sur la foi, l'Église, la pastorale, la communication, etc., ou qui justifient leurs choix stra- 
tégiques à partir aussi bien de données issues des sciences humaines ou des TP empiriques que des données de la tradition chrétienne.

\subsection{Dieu, sujet de l'histoire et Parole interpellante}

Sauf exceptions, les TP pastorales paraissent moins critiques quant au rapport épistémologique à Dieu que les TP fondamentales. Quand elle est le fait d'agents sur le terrain pastoral, la TP est confrontée à des exigences d'action et d'animation de la foi, à un monde de décision et à un univers épistémologique bien différent de l'univers universitaire. Dans ces TP pastorales, Dieu occupe habituellement une place maîtresse comme sujet de l'histoire, comme sujet rassemblant la communauté, comme sujet personnel interpellant la conscience et l'agir croyants. Leur rapport à Dieu, particulièrement dans les documents ecclésiaux, paraît donc plus proche de celui des TP critiques que des TP empiriques ou fondamentales. Par ailleurs, c'est souvent à partir des questions pastorales visant le monde aussi bien que l'Église que s'élaborent les TP empiriques, critiques et réflexives dont nous avons parlé plus haut. De la sorte, Dieu reste toujours présent en TP, ne serait-ce que comme une question ouverte que le théologien ou la théologienne adressent au monde aussi bien qu'à soi-même.

\section{La perplexité d'un constructiviste nostalgique ${ }^{18}$}

Alors que mes étudiants peuvent me considérer comme un constructiviste, des collègues me considèrent comme un réaliste ${ }^{19}$. Pas facile de faire la part des choses. Comme plusieurs, mon éducation théologique, assise sur une éducation religieuse de fondement métaphysique dans une culture empiriste, a été confrontée à divers tournants épistémologiques qui ne peuvent que marquer le rapport à Dieu : tournants empirique, existentiel, politique, linguistique, praxique, herméneutique, pragmatiste, constructiviste, etc. 20 En

18 J'emprunte le terme à une conférence d'Hans Ulrich GUMBRECHT à l'Université de Montréal en mars 1997.

19 Je songe ici à une boutade de Johannes VAN DER VEN.

20 Le tournant empirique sortait la théologie des bibliothèques pour la ramener à ce que certains appelaient "le pays réel»; le tournant existentiel ramenait la pensée au sujet et à son expérience; le tournant politique saisissait le croyant dans sa dimension sociale alors que le 
regard de cette culture et du savoir scientifique, on peut éprouver beaucoup de difficultés avec le concept traditionnel d'un Dieu personnel. Par ailleurs, en faire un symbole de notre relation à l'univers ou une construction psychosociale ne satisfait ni " les raisons sensibles " ni l'espérance du croyant. Le priant n'entend pas s'adresser à un concept mais à quelqu'un, fut-il "construit " (comme nous le sommes tous et toutes). D'où l'attrait du titre particulièrement signifiant de l'ouvrage de Richard Rorty : L'espoir au lieu du savoir.

Si l'empirisme classique rejette la prétention à la vérité du discours théologique qui ne porte pas sur des données vérifiables, si une perspective empirique naïve nie Dieu et marginalise la théologie, le pragmatisme et le constructivisme me semblent plus ouverts bien qu'ils signent aussi la fin de la référentialité. Ainsi, c'est en fonction de l'idée pragmatiste de la vérité que Viau tente de saisir la véracité du langage théologique et de l'établir en rapport à celle du langage scientifique. Le pragmatisme, en effet, considère la vérité non comme une adéquation au "réel " mais comme un processus qui se développe au contact de la "réalité ", comme le fait d'énoncés liés aux différentes opérations langagières d'un locuteur et produisant des fruits dans l'expérience (VIAU 1993 : 199). Dans une telle conception, la théologie (toujours pratique) s'avère progressive, liée à une expérience mouvante. Elle " affirme que les énoncés qui composent le discours qu'elle produit sont rendus vrais, se vérifient dans l'action et que ce ne sont plus, dès lors, des reflets d'une vérité absolue " (VIAU 1993 : 278). La vérité s'avère alors événement en travail, et le "faire la vérité " relève aussi bien de l'enquête que de l'interaction sociale.

tournant linguistique le montrait déterminé par le langage qu'il habitait, et que le tournant praxique le retrouvait agi et agissant; le tournant herméneutique amenait l'interprétation croyante à se faire consciente et critique d'elle-même et de ses processus; le tournant pragmatiste jaugeait la théologie à l'interaction entre les humains, alors que le tournant constructiviste intègre plusieurs de ces perspectives et redécouvre la théologie avec ses concepts, référents et perspectives comme construits historiques et culturels relevant de la même dynamique que les autres disciplines des sciences humaines. 
De son côté, le constructivisme paraît nier la réalité et s'approcher de l'idéalisme. Dieu s'y avère une "réalité " non seulement aussi construite (et efficace) que les autres, mais davantage même que bon nombre de concepts tels l'électricité, la fission de l'atome, la perte d'emploi ou la faim, dont les effets s'imposent. À la réflexion, cependant, le constructivisme ne paraît viable que sur la base d'un certain réalisme, fut-il mis entre parenthèse ou même dénié. Dire en effet qu'on ne peut atteindre la réalité, mais que notre construction de celle-ci est assez réussie pour y vivre ou que les concepts sont comme des sondes qu'on envoie dans la réalité pour l'explorer (SCHULTZ, LUCKMANN), cela n'est pas nier la réalité. C'est toutefois, et à l'évidence, s'y situer différemment. Déprendre la prise que nous avions ou que nous prétendions avoir sur elle.

Or la théologie affirme depuis longtemps que ce que nous disons de Dieu il faut aussitôt le dédire car il est toujours autre. Ou, au mieux, que nous ne pouvons parler de Dieu que par analogie. C'est ainsi que la théologie contemporaine, particulièrement la théologie américaine, a mis à l'honneur le rôle théologique de l'imagination dans ses fonctions poétiques et symboliques ainsi que dans son ouverture à la transcendance. La théologie, on l'a souvent dit, part de l'humain pour élaborer son discours. Or, celui-ci n'est pas qu'un être rationnel ou (de contrôle) technique, mais aussi un être corporel d'intersubjectivité, de symbolique et de transcendance.

Avant que les TP empirique et fondamentale considèrent Dieu comme objet de discours et de croyance, la théologie l'avait déjà objectivé en objet de connaissance et de transmission... dogmatiques. La sécularisation des sociétés occidentales et les sciences humaines l'ont évacué comme sujet de l'histoire, voire comme réfé rence valable. Mais si la puissance pratique et rationnelle de l'humanité a signé cette évacuation de Dieu, on sait que certains de nos concitoyens y reviennent, particulièrement quand ils font face à une impuissance, à une angoisse ou à un excès qui fait brèche à la rationalité dominante. Horkheimer (1994) signalait déjà que la foi en Dieu n'était pas d'abord le fait des fonctionnaires religieux ou politiques qui se la sont appropriée, mais celui des masses sans espoir terrestre. Plus récemment, les entrevues de John Horjan (The End of Science) avec des scientifiques de haut niveau le mènent à croire que ces scientifiques, sauf trois résolument athées ou agnostiques, portent dans leurs recherches une nostalgie religieuse ou y poursuivent en 
fait une quête religieuse. Les questions du sens ultime et du cheminement vers l'Être, que le rationalisme a laissées à l'herméneutique, à la métaphysique et à la théologie ${ }^{21}$, demeurent donc bien vivantes et signifiantes pour nos contemporains.

Weber (1964) considérait le sens qu'a une action pour son agent comme l'objet de recherche des sciences sociales, bien que ce sens ne soit pas sujet à observation empirique. Popper (1984:32 et 14) mettait en garde contre la tentation d'identifier « n'appartenant pas à la science empirique " avec «dépourvu de sens ou de signification " et il précisait que les énoncés métaphysiques ne sauraient être dépourvus de signification simplement parce qu'ils ne sont pas scientifiques, mais ils ont à trouver ailleurs leur validité. C'est à la quête critique de cette validité pour une existence juste et sensée qu'œuvre la théologie pratique, hantée, selon le mot d'Horkheimer placé en exergue de ce texte, par la nostalgie de ceux qui espèrent et qui affirment ou appellent l'existence d'un Autre. À la condition que la théologie pratique, mais aussi les sciences humaines, continue de considérer sérieusement la validité et les limites de ses affirmations, « Dieu » pourra s'y déployer comme cet Autre dont la théologie pratique se veut discours vivifiant. 


\section{Bibliographie}

AUDINET, Jacques, 1977. "Théologie pratique et pratique théologique », dans Le déplacement de la théologie. Paris, Beauchesne, pp. 91-108; repris dans AUDINET (1995: 101-113).

AUDINET, Jacques, 1995. "Diversité des théologies pratiques", dans Écrits de théologie pratique. Ottawa-Paris-Bruxelles-Genève, Novalis-Cerf-Lumen Vitae-Labor et Fides, 1995, pp. 239-358; d'abord publié dans Revue de l'Institut catholique de Paris 33 (1990) 75-96, et dans J. DORÉ (dir.), Introduction à l'étude de la théologie, tome 2. Paris, Desclée, 1990, pp. 541-574.

BARONI, Lise, Y. BERGERON, P. DAVIAU et M. LAGÜE, 1995. Voix de femmes, voies de passage. Pratiques pastorales et enjeux ecclésiaux. Montréal, Paulines.

BOFF, Clodovis, 1990. Théorie et pratique. Paris, Cerf.

BROWNING, Don S., 1991. A Fundamental Practical Theology. Minneapolis, Fortress.

BROWNING, Don S., dir., 1983. Practical Theology. The Emerging Field in Theology, Church, and World. San Francisco, Harper and Row.

CAMPBELL, Alastair V., 1990. "The Nature of Practical Theology ", dans D. B. FORRESTER, ed., Theology and Practice. London, Epworth Press, pp.10-20.

CASALIS, Georges, 1973. "Théologie pratique et pratique de la théologie ", dans Orientations. Paris, Beauchesne, pp. 85-105.

DAVIDSON, Donald, 1993. Enquêtes sur la vérité et l'interprétation. Nimes, Editions Jacqueline Chambon.

DUQUOC, Christian, 1996. Article «Théologie», dans l'Encyclopédie Catholicisme, t. 68. Paris, Letouzey et Ané.

EICHER, Peter, 1982. La théologie comme science pratique. Paris, Cerf.

FORTIN-MELKEVICK, Anne, 1994. "Les méthodes en théologie.

La pensée interdisciplinaire ", Concilium 256:131-142.

GARCEAU, Benoît, 1989. «Les défis des sciences humaines pour l'intelligence de la foi », dans J. CROTEAU, dir., Défis présents et 
à venir de l'université catholique. Ottawa, Université Saint-Paul, pp. 139-166.

GEFFRÉ, Claude, 1983. Le christianisme au risque de l'interprétation. Paris, Cerf.

GESCHÉ, Adolphe, 1995. "La théologie dans le temps de l'homme ", dans Association européenne de théologie catholique, Cultures et théologies en Europe. Paris, Cerf, pp. 109-142.

GISEL, Pierre, 1975. Vérité et Histoire. La théologie dans la modernité. Ernst Käsemann. Paris-Genève, Beauchesne-Labor et Fides.

GREINACHER, Norbert, 1990. "La théologie pratique en tant que théorie critique de la pratique ecclésiale dans la société ", dans A. VISSCHER, dir., Les études pastorales à l'université. Ottawa, Presses de l'Université d'Ottawa, pp. 279-297.

GROOME, Thomas H., 1976. «Le partage de la praxis chrétienne ", Lumen Vitae $31 / 1: 61-86$.

GROOME, Thomas H., 1991. Sharing Faith. A Comprehensive Approach to Religious Education and Pastoral Ministry. San Francisco, Harper.

GRÖZINGER, Albrecht, 1987. Praktische Theologie und Ästhetik : ein Beitrag zur Grundlegung der praktischen Theologie. Munich, Chr. Kaiser.

HEIDEGGER, Martin, 1969. "Phénoménologie et théologie " (conférence au Cercle de Théologie évangélique de Tübingen, le 9 juillet 1927), Archives de Philosophie XXXII : 357-395.

HEWITT, Mary A., 1995. Critical Theory of Religion. A Feminist Analysis. Minneapolis, Fortress Press.

HORKHEIMER, Max, 1978 Théorie critique. Paris, Payot.

HORKHEIMER, Max, 1994, Crépuscule : notes en Allemagne (19261931). Paris, Payot.

JÜNGEL, Eberhard, 1968. Die Praktische Theologie zwischen Wissenschaft und Praxis. Munich, Kaiser.

KERR, Fergus, 1991. La théologie après Wittgenstein. Paris, Cerf. 
KINAST, Robert, 1983. "Orthopraxis, Starting Point for Theology ». Catholic Theological Society of America Proceedings 38, pp. 29-44.

LALIVE D'EPINAY, C., 1978. " De l'impertinence. Un sociologue parmi les théologiens", dans J.J. von ALLMEN et all., Communion et communication. Genève, Labor et Fides, pp. 83-93.

LUCIER, Pierre, 1987. "Théologie et praxéologie", dans J.-G. NADEAU, dir., La praxéologie pastorale tome 1. Montréal, Fides, pp. 15-31. D'abord publié dans Études pastorales '74. Faculté de théologie, Université de Montréal (1974) pp. 27-40.

MÉNARD, Camil, 1992. «L'urgence d'une théologie pratique nordaméricaine comme théorie critique de l'agir chrétien au service de la société ", dans J.C. PETIT et J.-C. BRETON, dir., Seul ou avec les autres? Le salut chrétien à l'épreuve de la solidarité. Montréal, Fides, pp. 297-318.

METTE, Norbert, 1978. Theorie der Praxis : wissenschaftsgeschichtliche und methodologische Untersuchungen zur Theorie-Praxis-Problematik innerhalb der praktischen Theologie. Düsseldorf, Patmos-Verlag.

METTE, Norbert, 1979. "Praktische Theologie als Handlungswissenchaft ", Diakonia 10:190-203.

MOORE, Mary-Elizabeth, 1995. The Aims of Practical Theology : Diversity in the United States, manuscrit.

NADEAU, Jean-Guy, 1990. «Pour une science de l'action pastorale. Le souci de la pertinence pratique ", dans A. VISSCHER, dir., Les Études pastorales à l'université - Pastoral Studies in a University Setting. Ottawa, Presses de l'Université d'Ottawa, pp. 136-150.

NADEAU, Jean-Guy, 1993a. «Faire théologie selon un paradigme praxéologique », Théologiques $1 / 1: 79-100$.

NADEAU, Jean-Guy, 1993b. «La fonction révélante des pratiques pastorales », dans B. REYMOND et J.-M. SORDET, dir., La théologie pratique. Statut, méthodes, perspectives d'avenir. Paris, Beauchesne, pp. 103-116.

NADEAU, Jean-Guy, 1994a. " Dire Dieu dans les pratiques pastorales, une fonction de l'imagination? ", dans C. MÉNARD et F. VILLENEUVE, dir., Dire Dieu aujourd'hui. Montréal, Fides, pp. 239-253. 
NADEAU, Jean-Guy, 1994b. "Le témoignage chez les Alcooliques Anonymes: une pratique communicationnelle religieuse, " Studies in Religion/Sciences Religieuses, 23/4 : 469-484.

NADEAU, Jean-Guy et Hélène FOUCAULT, 1992. "Évaluation pastorale, recherche évaluative et autorité des approches empiriques en théologie pratique ", dans M. PELCHAT, dir., Les approches empiriques en théologie. (Coll. Théologies pratiques 4), Faculté de théologie, Université Laval, pp. 51-78.

NADEAU, Jean-Guy et Marcel VIAU, 1996. "La nouvelle théologie pratique : un débat ", Studies in Religion/Sciences Religieuses 25/2: 215-225.

NIPKOW, Karl Ernst, 1993. «Empirical Research within Practical Theology », Journal of Empirical Theology vol 6, no $1: 50-63$.

OGLETREE, Thomas W, 1983. « Dimensions of Practical Theology : Meaning, Action, Self », dans Don S. BROWNING, dir, Practical Theology. San Francisco, Harper and Row, pp. 83-101.

PEUKERT, Helmut, 1976. Wissenschaftstheorie - Handlungstheorie Fundamentale Theologie. Dusseldorf. Trad. : Science, Action, and Fundamental Theology. Toward a Theology of Communicative Action. Cambridge (Mass) et London (England), MIT Press, 1984.

POLING, James. N. et Donald E. MILLER, 1985. Foundations for a Practical Theology of Ministry. Nashville, Abingdon.

POPPER, Karl R., 1984. La logique de la découverte scientifique. Paris, Payot.

RAHNER, Karl, 1970. «Problèmes de théologie pratique » (Die praktische Theologie im Ganzen der theologischen Disziplinen, 1968), Écrits théologiques 11, Brughes, DDB-Mame, pp 109-127.

REYMOND, Bernard et Jean-Michel SORDET, dir., 1993. La théologie pratique. Statut, méthodes, perspectives d'avenir. Paris, Beauchesne.

RICOEUR, Paul, 1977. "Herméneutique de l'idée de Révélation ", dans P. RICGEUR et all., $L a$ révélation. Bruxelles, Facultés universitaires Saint-Louis, pp. 15-54.

RORTY, Richard, 1995. L'espoir au lieu du savoir. Introduction au pragmatisme. Paris, Albin Michel. 
SCHLEIERMACHER, Friedrich, 1988. Christian Caring, Selections from 'Practical Theology". Philadelphia, Fortress Press.

SCHLEIERMACHER, Friedrich, 1994. Bref exposé des études de théologie (1810 et 1830). Genève, Labor et Fides.

TILLICH, Paul, 1967. Systematic Theology. Chicago, University of Chicago Press.

van der VEN, Johannes, 1988. "Practical Theology : from Applied Theology to Empirical Theology ", Journal of Empirical Theology $1 / 1: 7-28$.

van der VEN, Johannes, 1990. "Practical Theology as Critical Empirical Theology ", dans A. VISSCHER, dir., Les Études pastorales à l'université - Pastoral Studies in a University Setting. Ottawa, Presses de l'Université d'Ottawa, pp. 238-262.

van der VEN, Johannes, 1993, Practical Theology. An Empirical Approach. Kampen (The Netherlands), Kok Pharos.

van der VEN, Johannes, 1994, "L'approche empirique en théologie pratique ", Théologiques 2/1:109-125.

VIAU, Marcel, 1987. Introduction aux études pastorales. Montréal, Paulines.

VIAU, Marcel, 1993. La nouvelle théologie pratique. Montréal, Paulines; Paris, Cerf.

VISSCHER, Adrian, dir., 1990. Les Études pastorales à l'université Pastoral Studies in a University Setting. Ottawa, Presses de l'Université d'Ottawa.

WEBER, Max, 1964. The Theory of Social and Economic Organisation. New York, Free Press.

ZAHRNT, Heinz, 1971. Dieu ne peut pas mourir. Contre les fausses alternatives dans l'Église et la société. Paris, Cerf.

ZULEHNER, Paul, 1988. Article "Théologie pratique", dans P. EICHER, dir., Dictionnaire de théologie. Paris, Cerf, pp. 790-795. 